\title{
Developing a real-time PCR assay based on multiplex high- resolution melt curve analysis: $A$ pilot study in detection and discrimination of soil-transmitted helminth and schistosome species
}

\begin{tabular}{|r|l|}
\hline Journal: & Parasitology \\
\hline Manuscript ID & PAR-2018-0132.R2 \\
\hline Manuscript Type: & Research Article \\
\hline Date Submitted by the Author: & n/a \\
\hline Complete List of Authors: & $\begin{array}{l}\text { Cunningham, Lucas; Liverpool School of Tropical Medicine, Parasitology } \\
\text { Stothard, J.; Liverpool School of Tropical Medicine, 2Department of } \\
\text { Parasitology } \\
\text { Osei-Atweneboana, Mike; Council for Scientific and Industrial Research, } \\
\text { Environmental Biology and Health } \\
\text { Armoo, Samuel; Council for Scientific and Industrial Research, } \\
\text { Environmental Biology and Health } \\
\text { Verweij, Jaco; Elisabeth-TweeSteden Ziekenhuis, Microbiology and } \\
\text { Immunology } \\
\text { Adams, Emily; LSTM, Parasitology }\end{array}$ \\
\hline Key Words: & $\begin{array}{l}\text { Schistosomiasis, Soil-transmitted helminths, Disease surveillance, } \\
\text { Monitoring and evaluation, Real-time PCR, Melt curve analysis, NTD, HRM, } \\
\text { DNA }\end{array}$ \\
\hline
\end{tabular}


1 Developing a real-time PCR assay based on multiplex high-resolution melt curve analysis: A pilot

2 study in detection and discrimination of soil-transmitted helminth and schistosome species

3 Lucas J. Cunningham ${ }^{1}$, J. Russell Stothard ${ }^{1}$, Mike Osei-Atweneboana ${ }^{2}$, Samuel Armoo ${ }^{2}$, Jaco J.

4 Verweij $^{3}$ and Emily R. Adams ${ }^{1}$

$5{ }^{1}$ Department of Parasitology, Liverpool School of Tropical Medicine, Liverpool, L3 5QA, UK; ${ }^{2}$

6 Department of Environmental Biology and Health, Council for Scientific and Industrial Research -

7 Water Research Institute, P.O. Box M 32, Accra 102001, Ghana ${ }^{3}$ Laboratory for Medical

8 Microbiology and Immunology, Elisabeth Tweesteden, Tilburg, the Netherlands. 
With the push towards control and elimination of soil-transmitted helminthiasis and schistosomiasis in low and middle-income countries, there is a need to develop alternative diagnostic assays that complement the current in-country resources, preferably at a lower cost. Here, we describe a novel high-resolution melt-curve assay with six PCR primer pairs, designed to sub-regions of the nuclear ribosomal locus. Used within a single reaction and dye detection channel, they are able to discriminate Ancylostoma duodenale, Necator americanus, Strongyloides stercoralis, Ascaris lumbricoides, Trichuris trichiuria and Schistosoma spp. by high-resolution melt (HRM) curve analysis. Here we describe the primers and the results of a pilot assessment whereby the HRM assay was tested against a selection of archived faecal samples from Ghanaian children as characterised by Kato-Katz and real-time PCR analysis with species-specific TaqMan hydrolysis probes. The resulting sensitivity and specificity of the HRM was $80 \%$ and $98.6 \%$ respectively. We judge the assay to be appropriate in modestly equipped and resourced laboratories. This method provides a potentially cheaper alternative to the TaqMan method for laboratories in lower resource settings. However, the assay requires a more extensive assessment as the samples used were not representative of all target organisms.

Keywords: schistosomiasis, soil-transmitted helminthiasis, disease surveillance, monitoring and evaluation, real-time PCR, melt curve analysis, SCH, STH, HRM, DNA 
51 Soil-transmitted helminthiasis (STH) and schistosomiasis ( $\mathrm{SCH}$ ) are grouped within the neglected tropical diseases (NTDs); each disease can cause chronic suffering, and in many low and middleincome countries is often linked to poverty (Hotez et al., 2006). With the announcement of the London Declaration on NTDs and most recently the sustainable development goals, international attention directed at control of these diseases has grown (WHO, 2018). Control of STH and SCH is based upon preventive chemotherapy, typically by mass drug administration (MDA) campaigns, offering donated anthelmintic medicines to children attending school (Weatherhead et al., 2017).

Although STH and SCH infections can be found in the majority of community members, disease control programmes usually examine school age children as an indicator for the wider community to determine the presence or absence of each disease. This in turn directs and informs the control programme for the most appropriate MDA schedule, its frequency and if further disease surveillance is necessary. For intestinal helminths (i.e. STH and Schistosoma mansoni infections), field-based surveillance typically makes use of the Kato-Katz method; a low cost semi-quantitative faecal concentration method (Katz et al., 1972). Owing to its affordability and scalability, the method is recommended by WHO as an operational diagnostic, however, it is an imperfect standard due to lack of sensitivity, and multiple stool samples should be taken; it is not the parasitological method of choice for diagnosis of strongyloidiasis (Barenbold et al., 2017; Kongs et al., 2001; Turner et al., 2017).

In line with the need to develop more sensitive detection methods for STH and SCH alongside the continued use of Kato-Katz, alternative biomarkers have been investigated (Stothard, 2009; Stothard et al., 2014). These largely rely upon detection of parasite-specific DNA with realtime PCR and Taqman probes (Meurs et al., 2015). The need for more sensitive diagnostics is particularly pertinent in light infection intensity and low transmission settings for the Kato-Katz method becomes more misleading than informative due to low sensitivity (Al-Shehri et al., 2018). Nonetheless novel methods also need to be both practical and cost-effective (Montresor et al.; Savioli et al., 2015). A drawback of multiplex TaqMan probe technology, for example, is the expense per reaction and need for thermal cycling machines with four or more reporting dye detection channels. In more modestly resourced laboratories, such machines are often not available whereas the technology entry standard, a two-dye channel machine, is available. 


\section{METHODS}

\section{Primer design}

The sequence for the nuclear ribosomal internal transcribed spacer (ITS) region or small sub-unit (18S) region for Ancylostoma duodenale, Necator americanus, Strongyloides stercoralis, Ascaris lumbricoides, Trichuris trichiuria, Schistosoma haematobium and Schistosoma mansoni was downloaded from the NCBI data base and aligned on Mega 6 (MEGA). Areas of high variability were then selected and entered into Primer 3 in order to generate primers and products with a specific melting temperature amenable for melt curve analysis. These primers were then checked for cross reactivity using NCBI Primer 3 primer design tool. Species-specific primers were designed for each helminth except for S. mansoni and S. haematobium where a generic primer pair was designed. The primers, see Table 1, were checked for specificity using the NCBI Primer 3 tool and cross-checked against the NCBI genomic data base.

\section{Optimisation and assessment of primers}

The primers were optimised using an annealing temperature gradient (Bio-Rad Chromo 4) from $50^{\circ} \mathrm{C}$ to $65^{\circ} \mathrm{C}$, this allowed for the assessment of which temperature the primers performed optimally at, as there may be differences between primers as to which temperatures they can function in. Similarly to the temperature gradient the efficiency of the primers was assessed using a primer limiting assay with a primer concentration range of $50 \mathrm{nM}, 100 \mathrm{nM}, 200 \mathrm{nM}$ and $300 \mathrm{nM}$. The importance of establishing the optimum primer concentration to use is based on the possibility that there may be differences in copy number and efficiency between the different primer pairs. The identification of the minimum amount of primer required is essential when running a multiplex reaction to ensure one primer pair does not out-compete others, as the resources in each reaction are finite. The primers were tested and optimised as single plex and later as a multiplex on two qPCR machines, the Chromo 4 (Bio-Rad Technologies) and Rotor Gene (Qiagen). To optimise the primers, clinical samples that had been identified as positive by TaqMan for S. stercoralis, N. americanus and A. duodenale were tested. For A. lumbricoudes, T. trichiuria and Schistosoma s.p., DNA was extracted from LSTM collections of whole worms stored in $100 \%$ ethanol. These worms had been previously collected from endemic regions around Lake Albert, Uganda, to supplement the materials used in the teaching department at the Liverpool school of Tropical Medicine. DNA was extracted by a rapid boil-and-spin method. In brief $\sim 0.2 \mathrm{~g}$ of worm tissue was isolated and washed three times in distilled water to remove the ethanol. Following the wash steps the worm tissue was placed in $200 \mu \mathrm{l}$ of TE buffer with an addition of $25 \mu \mathrm{l}$ of proteinase $\mathrm{K}(20 \mathrm{mg} / \mathrm{mL})$. The samples were then incubated at $55^{\circ} \mathrm{C}$

4| P a g e 
115 for two hours followed by an enzyme denaturation step of $90^{\circ} \mathrm{C}$ for 10 minutes. The samples were 116 vortexed and spun down and approximately $180 \mu \mathrm{l}$ of supernatant was removed and stored at $-20^{\circ} \mathrm{C}$.

\section{Assessment of field samples}

118

Having optimised the HRM primers and tested them in a multiplex assay, they were then tested on the DNA extracts of 32 faecal, samples that had been collected in 2017; as part of an ongoing longitudinal study screening for STH and SCH infections in Ghana. As part of the study, the samples had been screened using both Kato-Katz (two slides per faecal sample). Later the samples underwent a specific faecal DNA extraction method that incorporated a bead-beating stage, to mechanically rupture the helminth eggs, allowing for the DNA to be more reliably extracted. This method has previously been described (Cunningham et al., 2018). Following the DNA extraction process the samples underwent a pentaplex TaqMan assay that targeted Hookworm (Verweij et al., 2007), Schistosoma s.p.(Obeng et al., 2008), S. stercoralis (Verweij et al., 2010), T. trichiuria (Liu et al., 2013) and A. lumbricoides (Wiria et al., 2010).

\section{Thermal cycling conditions}

Optimisation

The following thermal cycle times were used, $95^{\circ} \mathrm{C}$ for $30 \mathrm{~s}$ followed by 35 cycles of $95^{\circ} \mathrm{C}$ for $15 \mathrm{~s}$ and a temperature gradient $50^{\circ} \mathrm{C}-65^{\circ} \mathrm{C}$ for $15 \mathrm{~s}$. The melt curve ramped from $65^{\circ} \mathrm{C}$ to $94^{\circ} \mathrm{C}$, rising by $0.5^{\circ} \mathrm{C}$ with a wait of 3 seconds. The supermix used was the SsoAdvanced ${ }^{\mathrm{TM}}$ universal SYBR ${ }^{\circledR}$ Green Supermix from Bio-Rad and the primers were run in a single plex reaction at a final concentration of $250 \mathrm{nM}$ in a $20 \mu \mathrm{L}$ reaction with $10 \mu \mathrm{L}$ of supermix and $2 \mu \mathrm{L}$ of DNA.

\section{Testing of field samples}

For the testing of the field samples the Type-it HRM PCR kit from Bioline was used with an initial melt step of $95^{\circ} \mathrm{C}$ for five minutes followed by 40 cycles of $96^{\circ} \mathrm{C}$ for $10 \mathrm{~s}, 60^{\circ} \mathrm{C}$ for $30 \mathrm{~s}$ and $72^{\circ} \mathrm{C}$ for 10s. The HRM ramped from $60^{\circ} \mathrm{C}$ to $95^{\circ} \mathrm{C}$, rising by $0.1^{\circ} \mathrm{C}$ every two seconds. The difference in use of supermix used in the optimisation and testing of field samples resulted from the development process whereby the initial intention was to design the primers for use with SYBR green for a twotube assay. Having optimised them they were then tested with the Type-it HRM kit to be run as a single tube assay with the field samples.

\section{RESULTS}

Performance of assay 
$60^{\circ} \mathrm{C}$ was chosen as the optimum annealing temperature; during primer limiting assessment as all primers performed equally well at this temperature. All primers also performed optimally at $200 \mathrm{nM}$, with the exception of the primers for Schistosoma which were found to be able to operate best at $100 \mathrm{nM}$. When tested against non-target species it was found that the primers were highly specific and did not cross react with non-target DNA.

<Insert Table 1 here>

The final primer selection is given in table 1, alongside their respective Tm and product size and melt temperatures. The positions of each primer pair on the nuclear ribosomal DNA of the different target helminths is shown in a diagrammatic form in Fig. 1.

<Insert Fig. 1 here>

The primers were tested as a multiplex reaction to determine the spread of the different melt peaks with each target DNA tested in triplicate. The distribution of the melt peaks is shown below in Fig. 2 . The results show that the products amplified for each helminth species have a distinct Tm to allow for separation of amplicons using the melt analysis process. There are also no other non-specific peaks that would indicate cross reaction between the different primers and non-specific DNA targets.

<Insert Fig. 2 here

\section{Analysis of field samples}

From the field survey a total of 32 faecal samples were examined using the six-primer pair set, these samples were positive by the TaqMan assay for hookworm, Schistosoma sp. and S. stercoralis; they did not contain positive samples for $T$. trichiuria and $A$. lumbricoides. The prevalence of the three helminth types present in the samples is given in Table 2.

<Insert table 2 here>

6 | P a g e 
174 The sensitivity and specificity of the new HRM assay was assessed against the TaqMan assay for all

175 helminth positives, resulting in a sensitivity of $80 \%$ and a specificity of $98.6 \%$, (Table 3.).

176

177

178

179

180

181

182

183

184

185

186

187

188

189

190

191

192

193

194

195

196

197

198

199

200

201

202

203

204

205

206

<Insert table 3 here>

\section{DISCUSSION}

Here we developed a novel HRM assay for the detection of STH and SCH our results show that the new primers are capable of discriminating between the different helminth species being targeted.

Developing diagnostic assays appropriate for use in modestly equipped and financed laboratories will expand the outreach of molecular surveillance approaches for STH and $\mathrm{SCH}$. While traditional PCR methods can be both sensitive and specific they require relatively long post amplification analysis phases (e.g. gel electrophoresis) which increases the risk of cross-contamination. By using real time PCR detection technology with TaqMan hydrolysis probes both hurdles are overcome but although such assays are highly sensitive and specific, they are more expensive in both consumables and in the type of real time PCR machine required for multiplexing, i.e. an increasing number of light channels receptors. The initial cost of the probes used in the TaqMan assay was a total of $f 1087$, at a concentration of $20 \mathrm{nmol}$. Depending on the volumes of probe used per reaction this will equate to an addition of $\sim 18 p$, per-probe, per-reaction adding a total of $\sim f 1.00$ per reaction. In this study the total cost per reaction for a single tube assay was $£ 2.13$ for a TaqMan reaction and $£ 0.75$ for an HRM reaction. If the number of light channels in the qPCR platform is less than six or five, then it would be necessary to run the TaqMan as a two-tube assay, doubling the cost of the reaction buffer required and resulting in a cost of $£ 3.05$ per sample. However, it is important to note that the initial cost of the DNA extraction process would remain the same for both assays. The cost of the DNA extraction and purification process exceeds that of either the cost of the TaqMan assay or the HRM assay and equates to approximately $f 5-f 6$ per sample.

In the present assay amplicons were designed to have characteristic melt profiles, based on sequence length (i.e. number of bases) and the base-pair composition (i.e. the relative proportion of purine and pyrimidine inter-strand bonding). Since the intercalating dye only fluoresces when bound to double stranded DNA, the melting point (where the double stranded DNA disassociates into single strands) is witnessed by a characteristic sharp drop in fluorescence which is detected by the machine and converted into a peak, see Fig. 2. The screening of the faecal samples showed an $80 \%$ correlation with the TaqMan method; although four positives identified by TaqMan were not detected with the HRM primers. A very plausable reason for this could be the low levels of target DNA within these samples as the TaqMan Ct values for these four samples ranged from 35-37 which are known to show low reproducibility in a TaqMan assay as well (Table 1, Appendices). Both

7| P a g e 
207 the TaqMan and HRM assays failed to detect the four Kato-Katz hookworm positives, the TaqMan 208 assay also failed to amplify seven of the Kato-Katz SCH positives and the HRM assay failed to detect 209 eight of the Kato-Katz positives. For the hookworm samples the low number of helminth eggs likely contributed to the two molecular methods failing to detect them. The $\mathrm{SCH}$ false negatives are harder to explain as only three samples missed, by both assays, would be considered light infections, the rest are all heavy infections. Failure to amplify DNA due to inhibitors is unlikely to be the reason as the TaqMan assay included an internal positive control that successfully amplified in all samples, within the expected $\mathrm{Ct}$ range. The heterogeneity of egg distribution within a faecal sample is a possible cause for the false negatives observed. If the stool sample had not been correctly mixed so as to more evenly distribute the eggs throughout then it is possible that the $\sim 0.1 \mathrm{~g}$ of faeces that underwent the DNA extraction process may have contained too few eggs for the assay to detect, despite high egg counts in the Kato-Katz readings.

The approach proposed in this paper capitalises on the sensitivity and specificity of the real time PCR platform but it only operates on a single channel, (Excitation $(\mathrm{nm}): \sim 470 \pm 10$, Detection $(\mathrm{nm}): \sim 510 \pm 5$ ), and does not require expensive probes. For these reasons it is much cheaper and can be used on all real time PCR machines that can detect this spectrum. This assay has been designed for use either with a high-resolution melt (HRM) kit and software or with SYBR Green and a standard melt-curve analysis setting. The use of SYBR would allow for this method to be used on a wider range of machines as not all have HRM capabilities, however it may require a two-tube assay approach to properly differentiate between the peaks as SYBR has less resolution than the HRM dye (EvaGreen). This new cheaper approach to screen for SCH and STH provides an alternative to the more expensive TaqMan approach which is pertinent to the capacity building of laboratories to screen for NTDs, and could make the adaptation of pre-existing infrastructures towards this aim more feasible.

The intention of this manuscript is to describe the initial results of a new diagnostic assay that may prove to be more attractive to molecular laboratories in low cost settings, due to dispensing with the need to buy expensive TaqMan probes. The assay can also work on less expensive qPCR thermocyclers, as it only requires a single channel to detect up to six target helminths. However, as previously mentioned the total cost including the DNA extraction protocol will equate to more than $f 5-f 6$ per sample, making the use of either qPCR method an expensive option for a diagnostic laboratory. To bring down the overall cost will require the development of a cheaper DNA extraction 
240 field of NTDs there were limited samples available for assay development resulting in a very limited 241 range of samples being used to run the validation assay. To fully assess the assay described in this 242 paper a wider range of positive samples will need to be screened to ensure that all target species are represented. This weakness in the study raises the importance of establishing biobanks, particularly 244 for NTDs, whereby samples can be stored and catalogued and made available to research groups. 245 Finally, the method described in this paper is limited to well relatively well-equipped laboratories 246 and requires highly trained technicians to carry out the assay. These factors make the assay 247 unsuitable for use in the field as a point of care test, of which there are already some available for 248 schistosomiasis notably the CCA rapid diagnostic test. Alternative, more field friendly molecular assays are also in development notably the isothermal methods such as LAMP (loop-mediated isothermal amplification) and RPA (recombinase polymerase amplification) have been used for the diagnosis of schistosomiasis (Fernandez-Soto et al., 2014; Rosser et al., 2015). These methods are more field friendly and depending on the nature of the sample, such as urine over faeces, may have simpler DNA isolation protocols. The multiplexing capabilities of these methods are often limited as well as the cost for the RPA assay being relatively higher compared to both LAMP and qPCR (Minetti et al., 2016).

Conclusion

To conclude we have developed a low-cost, potentially high through-put multiplex DNA assay useful for detection of STH and SCH. This assay could be an appropriate DNA detection technique in modestly equipped and resourced laboratories. Although current limitations to the study include the high cost of the DNA extraction protocol required and the need for a more comprehensive validation assessment.

Acknowledgements

The authors would like to thank DFID UK, the Liverpool School of Tropical medicine and the Council for Scientific and Industrial Research, Ghana for their support and contributions as well as Dr Thomas Edwards and Chris Williams for their technical advice.

\section{Financial support}

270 This work was supported by the Department for International Development (DFID) grant number PO 6407. 


\section{References}

Primer 3.

Al-Shehri, H., Koukounari, A., Stanton, M. C., Adriko, M., Arinaitwe, M., Atuhaire, A., Kabatereine, N. B. and Stothard, R. J. (2018). Surveillance of Intestinal Schistosomiasis During Control: A Comparison of Four Diagnostic Tests Across Five Ugandan Primary Schools in the Lake Albert Region. Parasitology.

Barenbold, O., Raso, G., Coulibaly, J. T., N'Goran, E. K., Utzinger, J. and Vounatsou, P. (2017). Estimating sensitivity of the Kato-Katz technique for the diagnosis of Schistosoma mansoni and hookworm in relation to infection intensity. Plos Neglected Tropical Diseases, 11. doi: 10.1371/journal.pntd.0005953.

Cunningham, L. J., Odoom, J., Pratt, D., Boatemaa, L., Asante-Ntim, N., Attiku, K., Banahene, B., Osei-Atweneboana, M., Verweij, J. J., Molyneux, D., Stothard, R. J. and Adams, E. R. (2018). Expanding molecular diagnostics of helminthiasis: Piloting use of the GPLN platform for surveillance of soil transmitted helminthiasis and schistosomiasis in Ghana. Plos Neglected Tropical Diseases, 12. doi: 10.1371/journal.pntd.0006129.

Fernandez-Soto, P., Arahuetes, J. G., Hernandez, A. S., Aban, J. L., Santiago, B. V. and Muro, A. (2014). A Loop-Mediated Isothermal Amplification (LAMP) Assay for Early Detection of Schistosoma mansoni in Stool Samples: A Diagnostic Approach in a Murine Model. Plos Neglected Tropical Diseases, 8. doi: 10.1371/journal.pntd.0003126.

Hotez, P., Ottesen, E., Fenwick, A. and Molyneux, D. (2006). The neglected tropical diseases: The ancient afflictions of stigma and poverty and the prospects for their control and elimination. Hot Topics in Infection and Immunity in Children lii, 582, 23-33.

Katz, N., Chaves, A. and Pellegrino, J. (1972). A Simple Device for Quantitative Stool thick-Smear Technique in Schistosomiasis mansoni. Rev Inst Med Trop Sao Paulo, 14, 397-400.

Kongs, A., Marks, G., Verle, P. and Van der Stuyft, P. (2001). The unreliability of the Kato-Katz technique limits its usefulness for evaluating S-mansoni infections. Tropical Medicine \& International Health, 6, 163-169. doi: 10.1046/j.1365-3156.2001.00687.x.

Liu, J., Gratz, J., Amour, C., Kibiki, G., Becker, S., Janaki, L., Verweij, J. J., Taniuchi, M., Sobuz, S. U., Haque, R., Haverstick, D. M. and Houpt, E. R. (2013). A Laboratory-Developed TaqMan Array Card for Simultaneous Detection of 19 Enteropathogens. Journal of Clinical Microbiology, 51, 472-480. doi: 10.1128/jcm.02658-12.

MEGA Molecular Evolutionary Genetics Analysis Home Page. Vol. 2013.

Meurs, L., Brienen, E., Mbow, M., Ochola, E. A., Mboup, S., Karanja, D. M. S., Secor, W. E., Polman, K. and van Lieshout, L. (2015). Is PCR the Next Reference Standard for the Diagnosis of Schistosoma in Stool? A Comparison with Microscopy in Senegal and Kenya. Plos Neglected Tropical Diseases, 9. doi: 10.1371/journal.pntd.0003959.

Minetti, C., LaCourse, J., Reimer, L. and Stothard, R. (2016). Focusing nuceleic acid-based molecular diagnostics and xenomonitoring approaches for human helminthiases amenable to preventive chemotherapy. Parasitology Open, 2.

Montresor, A., Crompton, D. W. T., Hall, A., Bundy, D. A. P. and Savioli, L. Guidelines for the evaluation of soil-transmitted helminthiasis and schistosomiasis at community level. (ed. WHO).

Obeng, B. B., Aryeetey, Y. A., de Dood, C. J., Amoah, A. S., Larbi, I. A., Deelder, A. M., Yazdanbakhsh, M., Hartgers, F. C., Boakye, D. A., Verweij, J. J., van Dam, G. J. and van Lieshout, L. (2008). Application of a circulating-cathodic-antigen (CCA) strip test and realtime PCR, in comparison with microscopy, for the detection of Schistosoma haematobium in urine samples from Ghana. Annals of Tropical Medicine and Parasitology, 102, 625-633. doi: 10.1179/136485908x337490.

Rosser, A., Rollinson, D., Forrest, M. and Webster, B. L. (2015). Isothermal Recombinase Polymerase amplification (RPA) of Schistosoma haematobium DNA and 
oligochromatographic lateral flow detection. Parasites \& Vectors, 8. doi: 10.1186/s13071015-1055-3.

Savioli, L., Fenwick, A., Rollinson, D., Albonico, M. and Ame, S. M. (2015). An achievable goal: control and elimination of schistosomiasis. Lancet, 386, 739-739. doi: 10.1016/s01406736(15)61536-7.

Stothard, J. R. (2009). Improving control of African schistosomiasis: towards effective use of rapid diagnostic tests within an appropriate disease surveillance model. Transactions of the Royal Society of Tropical Medicine and Hygiene, 103, 325-332. doi: 10.1016/j.trstmh.2008.12.012.

Stothard, J. R., Stanton, M. C., Bustinduy, A. L., Sousa-Figueiredo, J. C., Van Dam, G. J., Betson, M., Waterhouse, D., Ward, S., Allan, F., Hassan, A. A., Al-Helal, M. A., Memish, Z. A. and Rollinson, D. (2014). Diagnostics for schistosomiasis in Africa and Arabia: a review of present options in control and future needs for elimination. Parasitology, 141, 1947-1961. doi: 10.1017/s0031182014001152.

Turner, H. C., Bettis, A. A., Dunn, J. C., Whitton, J. M., Hollingsworth, D., Fleming, F. M. and Anderson, R. M. (2017). Economic Considerations for Moving beyond the Kato-Katz Technique for Diagnosing Intestinal Parasites As We Move Towards Elimination. Trends in Parasitology, 33, 435-443. doi: 10.1016/j.pt.2017.01.007.

Verweij, J. J., Brienen, E. A. T., Ziem, J., Yelifari, L., Polderman, A. M. and Van Lieshout, L. (2007). Simultaneous detection and quantification of Ancylostoma duodenale, Necator americanus, and Oesophagostomum bifurcum in fecal samples using multiplex real-time PCR. American Journal of Tropical Medicine and Hygiene, 77, 685-690.

Verweij, J. J., Canales, M., Polman, K., Ziem, J., Brienen, E. A. T., Polderman, A. M. and van Lieshout, L. (2010). Molecular diagnosis of Strongyloides stercoralis in faecal samples using real-time PCR (vol 103, pg 342, 2009). Transactions of the Royal Society of Tropical Medicine and Hygiene, 104, 378-378. doi: 10.1016/j.trstmh.2010.02.010.

Weatherhead, J. E., Hotez, P. J. and Mejia, R. (2017). The Global State of Helminth Control and Elimination in Children. Pediatric Clinics of North America, 64, 867-877. doi: 10.1016/j.pcl.2017.03.005.

WHO (2018). Fact Sheets Relating to NTDs. Vol. 2018.

Wiria, A. E., Prasetyani, M. A., Hamid, F., Wammes, L. J., Lell, B., Ariawan, I., Uh, H. W., Wibowo, H., Djuardi, Y., Wahyuni, S., Sutanto, I., May, L., Luty, A. J. F., Verweij, J. J., Sartono, E., Yazdanbakhsh, M. and Supali, T. (2010). Does treatment of intestinal helminth infections influence malaria? Background and methodology of a longitudinal study of clinical, parasitological and immunological parameters in Nangapanda, Flores, Indonesia (ImmunoSPIN Study). Bmc Infectious Diseases, 10. doi: 10.1186/1471-2334-10-77. 
Table 1. Final primer selection for each parasite with details of amplicon product size and Tm.

\begin{tabular}{|c|c|c|c|c|}
\hline Primer name & Target species & Sequence (5'-3') & Product size (bp) & Product $\mathrm{Tm}\left({ }^{\circ} \mathrm{C}\right)$ \\
\hline $\begin{array}{l}\text { mcAd_F } \\
\text { mcAd_R }\end{array}$ & A. duodenale & $\begin{array}{l}\text { CTGAATGACAGCAAACTCGTTG } \\
\text { ATTGCAAATAACAGAAACATCGT }\end{array}$ & 100 & 79.4 \\
\hline $\begin{array}{l}\text { mcStrongy_F } \\
\text { mcStrongy_R }\end{array}$ & S. stercoralis & $\begin{array}{l}\text { GATCATTCGGTTCATAGGTCGAT } \\
\text { TACTATTAGCGCCATTTGCATTC }\end{array}$ & 105 & 81.7 \\
\hline $\begin{array}{l}\text { mcNa_F } \\
\text { mcNa_R }\end{array}$ & N. americanus & $\begin{array}{l}\text { TGCACGCTGTTATTCACTACG } \\
\text { TTGCAAATGACACATCCACA }\end{array}$ & 179 & 83.8 \\
\hline $\begin{array}{l}\text { mcSCH_F } \\
\text { mcSCH_R }\end{array}$ & Schistosoma spp. & $\begin{array}{l}\text { TGTCGTATGCCCTGATGGTG } \\
\text { CCGGATCGCTTCAACAGTGT }\end{array}$ & 180 & 85.1 \\
\hline $\begin{array}{l}\text { mcAscaris_F } \\
\text { mcAscaris_R }\end{array}$ & A. lumbricoides & $\begin{array}{l}\text { TAATAGCAGTCGGCGGTTTC } \\
\text { CTCCACCTTTCATCGCTACC }\end{array}$ & 208 & 86.8 \\
\hline $\begin{array}{l}\text { mcTrich_F } \\
\text { mcTrich_R }\end{array}$ & T. trichiuria & $\begin{array}{l}\text { ATTGGAGGGCAAGTCTGGTG } \\
\text { TGAAGAGCATCCAGGGCAAT }\end{array}$ & 179 & 88.0 \\
\hline
\end{tabular}


Table 2. Percentage positive results of hookworm, Schistosoma s.p. and S. stercoralis for the following diagnostic assays: Kato-Katz, TaqMan and HRM, (n=number).

\begin{tabular}{|c|c|c|c|c|}
\hline & & \multicolumn{3}{|c|}{ Assay conducted } \\
\hline & & Kato-Katz (n) & TaqMan (n) & HRM (n) \\
\hline & Hookworm & $12.5(4)$ & $6.3(2)$ & $6.3(2)$ \\
\hline Helminth type & Schistosoma sp. & $59.4(19)$ & $53.1(17)$ & 46.9 (15) \\
\hline & S. stercoralis & $0(0)$ & $3.1(1)$ & $0(0)$ \\
\hline
\end{tabular}


Table 3. Numbers for sensitivity and specificity of the HRM assay using TaqMan as the gold standard for all helminth positives

\begin{tabular}{|c|c|c|c|}
\hline & \multicolumn{2}{|c|}{ TaqMan } \\
\hline & & Positive (n) & Negatve (n) \\
\hline \multirow{2}{*}{ HRM } & Positive & 16 & 1 \\
\hline & Negative & 4 & 75 \\
\hline
\end{tabular}




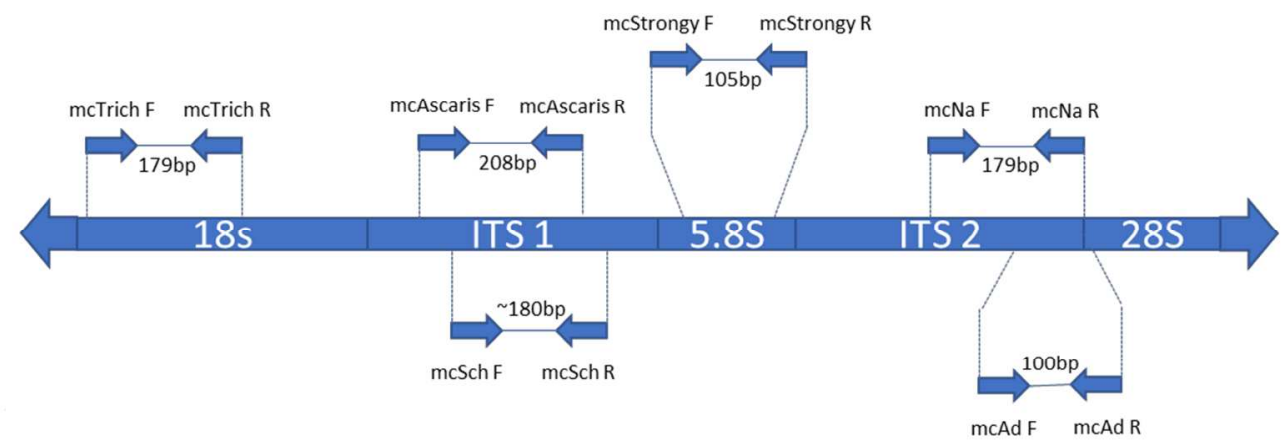

Fig. 1. Diagrammatic representation of the positions of the different primer pairs on the ribosomal DNA; "mc" stands for melt-curve.

$132 \times 52 \mathrm{~mm}(600 \times 600 \mathrm{DPI})$ 


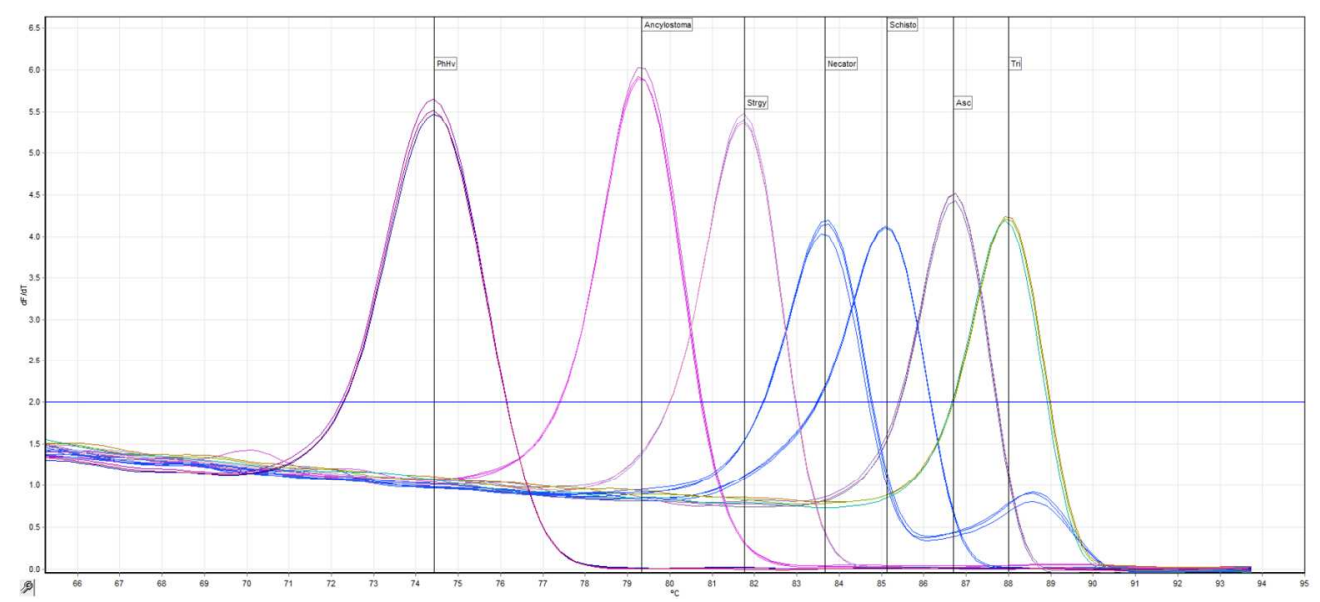

Fig. 2. A representative composite chromatogram of a single multiplex reaction showing the individual melt peaks for each primer pair and target parasite for: An. duodenale (Ad), N. americanus ( $\mathrm{Na}$ ), St. stercoralis (Strgy), As. lumbricoides (Asc), T. trichiuria (Tri) and Schistosoma s.p. (Sch).

$336 \times 153 \mathrm{~mm}(150 \times 150 \mathrm{DPI})$ 


\begin{tabular}{|c|c|c|c|c|c|c|c|c|c|c|c|c|c|}
\hline \multirow[b]{2}{*}{$\begin{array}{c}\text { Sample } \\
\text { No }\end{array}$} & \multicolumn{5}{|c|}{ Hookworm } & \multicolumn{5}{|c|}{ Schistosoma } & \multicolumn{3}{|c|}{ S. stercoralis } \\
\hline & TaqMan(Ct) & Kato-Katz & EPG & Intensity & HRM(Ct) & TaqMan(Ct) & Kato-Katz & EPG & Intensity & HRM(Ct) & TaqMan(Ct) & Kato-Katz & HRM(Ct) \\
\hline 1 & Pos (30) & 0 & & & Pos (30) & 0 & Pos & 72 & light & 0 & 0 & 0 & 0 \\
\hline 2 & Pos (33) & 0 & & & Pos (32) & 0 & Pos & 60 & light & 0 & 0 & 0 & 0 \\
\hline 3 & 0 & Pos & 36 & light & 0 & 0 & 0 & & & 0 & 0 & 0 & 0 \\
\hline 4 & 0 & Pos & 72 & light & 0 & 0 & 0 & & & 0 & 0 & 0 & 0 \\
\hline 5 & 0 & Pos & 36 & light & 0 & 0 & 0 & & & 0 & 0 & 0 & 0 \\
\hline 6 & 0 & Pos & 48 & light & 0 & Pos (34) & Pos & 336 & medium & Pos (30) & 0 & 0 & 0 \\
\hline 7 & 0 & 0 & & & 0 & Pos (25) & Pos & 528 & heavy & Pos (23) & Pos (37) & 0 & 0 \\
\hline 8 & 0 & 0 & & & 0 & Pos (24) & Pos & 360 & medium & Pos (23) & 0 & 0 & 0 \\
\hline 9 & 0 & 0 & & & 0 & Pos (31) & Pos & 816 & heavy & Pos (30) & 0 & 0 & 0 \\
\hline 10 & 0 & 0 & & & 0 & Pos (19) & Pos & 2424 & heavy & Pos (19) & 0 & 0 & 0 \\
\hline 11 & 0 & 0 & & & 0 & Pos (35) & Pos & 3948 & heavy & 0 & 0 & 0 & 0 \\
\hline 12 & 0 & 0 & & & 0 & Pos (25) & Pos & 264 & medium & Pos (26) & 0 & 0 & 0 \\
\hline 13 & 0 & 0 & & & 0 & Pos (29) & Pos & 780 & heavy & Pos (30) & 0 & 0 & 0 \\
\hline 14 & 0 & 0 & & & 0 & Pos (27) & Pos & 1152 & heavy & Pos (27) & 0 & 0 & 0 \\
\hline 15 & 0 & 0 & & & 0 & Pos (31) & Pos & 504 & heavy & Pos (31) & 0 & 0 & 0 \\
\hline 16 & 0 & 0 & & & 0 & Pos (35) & Pos & 528 & heavy & 0 & 0 & 0 & 0 \\
\hline 17 & 0 & 0 & & & 0 & Pos (30) & Pos & 216 & medium & Pos (29) & 0 & 0 & 0 \\
\hline 18 & 0 & 0 & & & 0 & Pos (37) & 0 & & & 0 & 0 & 0 & 0 \\
\hline 19 & 0 & 0 & & & 0 & Pos (27) & 0 & & & Pos (26) & 0 & 0 & 0 \\
\hline 20 & 0 & 0 & & & 0 & Pos (32) & 0 & & & Pos (33) & 0 & 0 & 0 \\
\hline 21 & 0 & 0 & & & 0 & Pos (31) & 0 & & & Pos (33) & 0 & 0 & 0 \\
\hline 22 & 0 & 0 & & & 0 & Pos (31) & 0 & & & Pos (32) & 0 & 0 & 0 \\
\hline 23 & 0 & 0 & & & 0 & 0 & Pos & 3612 & heavy & 0 & 0 & 0 & 0 \\
\hline 24 & 0 & 0 & & & 0 & 0 & Pos & 792 & heavy & 0 & 0 & 0 & 0 \\
\hline 25 & 0 & 0 & & & 0 & 0 & Pos & 3300 & heavy & Pos (24) & 0 & 0 & 0 \\
\hline 26 & 0 & 0 & & & 0 & 0 & Pos & 12 & light & 0 & 0 & 0 & 0 \\
\hline 27 & 0 & 0 & & & 0 & 0 & Pos & 432 & heavy & 0 & 0 & 0 & 0 \\
\hline 28 & 0 & 0 & & & 0 & 0 & 0 & & & 0 & 0 & 0 & 0 \\
\hline 29 & 0 & 0 & & & 0 & 0 & 0 & & & 0 & 0 & 0 & 0 \\
\hline 30 & 0 & 0 & & & 0 & 0 & 0 & & & 0 & 0 & 0 & 0 \\
\hline 31 & 0 & 0 & & & 0 & 0 & 0 & & & 0 & 0 & 0 & 0 \\
\hline 32 & 0 & 0 & & & 0 & 0 & 0 & & & 0 & 0 & 0 & 0 \\
\hline
\end{tabular}

